dition, ur steady motion, when any influences, distributed zonally, prevent a rotation with uniform average, angular velocity of the whole envelope, or at least of those parts that are at equal distances from the axis of rotation. If, therefore, such influences exist on the sun, then wave formations must occur on it, and the sun spots, which have already been considered as vortex phenomena, as well as their variability, would thus find an explanation similar to that of the cyclones and anticyclones of the terrestrial atmosphere. It thus becomes quite possible that the regular phenomena of the general atmospheric circulation of the earth may, for purely mechanical reasons, have the same period as those on the sun. In this case there would not need to exist any direct connection between the sun spots and our weather, even though the same period should be established beyond doubt by observation. On the other hand, a further development of the theory may, perhaps, lead to conclusions as to certain mechanical relations, especially as to the velocity of rotation of the sun.

Even the discussion as to the influence of the moon on our weather, which is still by no means settled, will, in this way, be brought to an end, for it must then be possible to satisfactorily show whether the otherwise regular processes of atmospheric circulation are influenced by the changes in the moon's position, especially by its motion in declination.

It is to be hoped that the method here indicated may lead meteorology out of the region of vacillating ideas that now control it into a broader field, and place it among the exact sciences, where everything is reduced to numerical computation, and thus, to an important extent, further its application to daily practice.

Deutsche Seewarte, Hamburg.

\title{
SOME POINTS IN THE ELEMENTS OF THE THEORY OF FUNCTIONS.
}

BY PROFESSOR W. F. OSGOOD.

I. A New Definition of an Analytic Function. Cauchy defined $f(z)$ to be an analytic function of $z$ when $f(z)$ is continuous and

$$
\frac{f(z+\Delta z)-f(z)}{\Delta z}
$$

converges toward one and the same limit when $\Delta z$ con- 
verges in any manner toward 0 . From this definition, coupled with a requirement concerning the derivative $f^{\prime}(z)$, the fundamental theorem of his whole theory is obtained, namely: If $f(z)$ is analytic and $f^{\prime}(z)$ continuous throughout a region, then $\int f(z) d z$ taken along any closed path lying in the region vanishes.

We may, however, equally well turn things about and take as definition the following:

DeFINITION : $f(z)$ is an analytic function of $z$ if $f(z)$ is continuous throughout a region and $\int f(z) d z$ taken along any closed path lying wholly within the region vanishes.

For then the function

$$
F(z)=\int_{a}^{z} f(z) d z
$$

will be an analytic function according to Cauchy's definition, and hence by Cauchy's theory its derivative $f(z)$ will also be analytic.

Thus while Cauchy's definition is based on the infinite process of differentiation, the present definition is based on the infinite process of integration, the integral being defined as the limit of a sum. Let it here be emphasized that these two processes, in the conceptions and definitions on which they rest, are wholly coördinate with each other; for neither can it be claimed in this respect that it is more elementary than the other.

There is one essential point of difference between these definitions. Cauchy's definition is based on the behavior of the function in a point; this definition prescribes the behaviour of the function throughout a region. Weierstrass's definition of an analytic function is of the latter kind. Furthermore, Cauchy is obliged in order to get any theorems worth mentioning, to demand $*$ that the derivative $f^{\prime}(z)$ be continuous throughout a region. Here, that requirement is brought nearer home by being transferred to the definition and there applies to the function itself.

It seems reasonable to expect that from this definition some elementary theorems will be more directly deducible than from Cauchy's definition. As an example take the theorem that a uniformly convergent series of analytic functions defines an analytic function. $\dagger$

\footnotetext{
* One may, if one pleases, incorporate this demand into the definition it seems to me undesirable to do so. But even then that which is essential in the present observation would not be changed.

$\dagger$ This application of the definition was called to my attention by Prof. Bôcher.
} 
Let the series be

$$
f_{1}(z)+f_{2}(z)+\cdots \cdots
$$

Then its value $f(z)$ is a continuous function $f(z)$ and it remains to show that $\int f(z) d z$ taken along any closed path vanishes. This follows at once from the uniform convergence; the series can be integrated term by term and the integral of each term along the path in question vanishes.

It is to be noticed that this proof belongs to the most elementary class of proofs, in that it calls for no explicit representation of the functions entering (e. g., by Cauchy's integral or by a power series).

II. A fundamental theorem in the elements of the theory of functions of a complex variable is the following:

(A) If $f(z)$ is single-valued and analytic at all points* of a certain region except at the point $z=c$, and if $f(z)$ does not become infinite at $c$, then $f(z)$ is analytic in $c$ also, or can be made so by the assignment to $f(z)$ in the point $c$ of the value that $f(z)$ approaches when $z$ approaches $c$.

This theorem forms the basis for the theorems relating to the behavior of a single valued function $f(z)$ in the neighborhood of a singular point, $P, f(z)$ being analytic at all points in the neighborhood of $P$, namely, either (1) the function

$$
\varphi(z)=(z-c)^{m} f(z), \quad z \neq c ; \quad \varphi(c)=0
$$

where $m$ denotes a positive integer, is analytic in $P$; or (2) $f(z)$ comes arbitrarily near to any assigned value in every neighborhood of the point $P$.

The proofs of this theorem ordinarily given are either wrong or far less elementary than one could wish, in view of the nature of the theorem. It is the purpose of this paper to consider some of these proofs and to give a new one which is at the same time simple and elementary.

Riemann's Proof.-Riemann $\dagger$ first proves the following lemma: Let $u$ be a harmonic function \$ at all points of a

* With the new definition of an analytic function given in I, by the expression " $f(z)$ is analytic in the point $a$ " is to be understood that $a$ lies within a region throughout which $f(z)$ is analytic.

† Inaugural Dissertation, Göttingen, 1851; each theorem is here stated in somewhat less general form than in the original.

$\ddagger$ By harmonic is meant a function which together with its first and second derivatives is continuous and satisfies Laplace's equation: $\Delta u=\frac{\partial^{2} u}{\partial x^{2}}+\frac{\partial^{2} u}{\partial y^{2}}=0$. 
region except $P$; let

$$
\lim _{\substack{x=0 \\ y=0}} \rho \frac{\partial u}{\partial x}=0, \quad \lim _{\substack{x=0 \\ y=0}} \rho \frac{\partial u}{\partial y}=0,
$$

where $\rho$ denotes the distance of the point $(x, y)$ from $P$; and finally let $u$ remain finite in the neighborhood of $P$; then $u$ is harmonic in $P$ also, or can be made so by assigning to $u$ in the point $P$ the value that $u$ approaches when $(x, y)$ approaches $P$ in any manner. He then states the theorem (A), replacing the words, "if $f(z)$ does not become infinite at $c$ " by " if $\lim (z-c) w=0$ when $z$ approaches $c "$, the function $f(z)$ being denoted by $w=u+v i$; and proves it as follows. He forms the integral

$$
U=\int_{\left(x 0, y_{0}\right)}^{(x, y)}(u d x-v d y)
$$

where $v$ denotes the conjugate function of $u$, shows that its value is independent of its path and that the function $U$ thus defined, which is continuous at $P$, is by the theorem just quoted harmonic at $P$ also. And since

$$
u=\frac{\partial U}{\partial x}, \quad v=-\frac{\partial U}{\partial y}
$$

the truth of the statement is evident.

The first of these theorems is interesting as showing how little stress Riemann laid on a systematic development of the theory of the logarithmic potential (i.e., of harmonic functions) as a basis for the theory of functions of a complex variable. From this point of view the theorem (A) would be deduced from the theorem (B): If $u$ is harmonic at all points of a certain region except at the point $P$ and if $u$ does not become infinite at $P$, then $u$ is harmonic in $P$ also, or can be made so by the assignment to $u$ in the point $P$ of its limiting value.

The Pseudo-Riemann Proof.-Riemann's rigorous proof of (A) has fared hardly with his expounders, whose version of it is as follows. Form the function

$$
\varphi(z)=(z-c) f(z), \quad z \neq c ; \quad \varphi(c)=0
$$

Then $\varphi(z)$ is analytic in the neighborhood of $c$ and continuous at $c$, therefore (sic) $\varphi(z)$ is analytic at $c$ and can be developed by Taylor's theorem :

$$
\varphi(z)=a_{0}+a_{1}(z-c)+a_{2}(z-c)^{2}+\cdots
$$


But $\varphi(c)=0$, hence $a_{0}=0$ and

or

$$
\begin{gathered}
\varphi(z)=(z-c) f(z)=a_{1}(z-c)+a_{2}(z-c)^{2}+\cdots \\
f(z)=a_{1}+a_{2}(z-c)+\cdots
\end{gathered}
$$

This proof was given in the second edition of Durège's Theorie der Functionen, 1873, and has not been changed in the translation that has just appeared of the fourth edition. Curiously enough the proof given in the first edition, 1864, was rigorous, or at least could readily have been made so,it is not complete. It was the proof given below as the first elementary proof and it seems likely that this proof was the one given by Riemann in his lectures. But in the improvements made in the second edition it was replaced by the one just cited. Harnack, whose desire frequently exceeded his ability to be rigorous, gave a similar proof,* likewise uncorrected in the English translation. Forsyth has also fallen into this error. $\dagger$ Biermann, in his attempt to expound Weierstrass's theory of functions, gives this same proof; $\$$ this passage was sharply criticised in the Fortschritte d. Math., vol. 19, 1887, p. 363.

Perhaps the reason for Durège's changing to the above proof is to be found in a paper by Roch, one of the earliest expounders of Riemann, which formed the second of a set of three papers on Riemann's theory, appearing in Schlömilch. $\dagger$ In $\$ 8$ (vol. 8, p. 187) Roch expounds \$13 of Riemann's inaugural dissertation and there does one of two things : either he makes precisely the error above pointed out or he tacitly assumes Theorem (A). On some accounts the latter seems more likely to be the case; but this passage would easily account for Durège's proof.

Proof by Laurent's Theorem. This theorem says that if $f(z)$ is analytic at all points of a circular ring, $i$. e., of a region included between two concentric circles with centres at $c$, then $f(z)$ can be expressed within this region in the form :

$$
f(z)=\sum_{-\infty}^{\infty} a_{\mu}(z-c)^{\mu}
$$

From this theorem the theorem (A) can be deduced. But this proof of (A), if given by Cauchy's methods, involves in addition to Cauchy's integral theorems the representation

* Diff.- u. Integralrechnung, \& 189.

†Theory of Functions, p. 52.

$\dagger$ Analytische Functionen, p. 174.

†† Zeitschrift f. Math. u. Phys. vol. 8, pp. 12-26 and pp. 183-203, 1863 and vol. 10, pp. 169-194, 1865. 
of $f(z)$ by a power series and some properties of such series; if given by Weierstrass's methods, is still less elementary, the establishment of Laurent's theorem in that theory coming at a comparatively late stage. This subject is discussed at some length by Pringsheim in the last number of the Math. Annalen, who develops a number of elementary theorems, inclusive of the theorem (A), by the aid of power series plus the infinite process known as the mean value, a process which is essentially a restricted form of integration, and thus combining with the method of power series that which is essential in complex integration for the theorems in hand, he gives Cauchy's proofs of theorems in power series without the aid of Cauchy's integral.

Elementary Proofs of $(A)$.- First Proof. Let $C$ denote any curve enclosing the point $c$ and lying within the region in question, and let $t$ be any point but $c$ within $C$. Surround $c$ by a small circle $\gamma$ not including $t$. Then by Cauchy's theorem

$$
f(t)=\frac{1}{2 \pi i} \int_{c} \frac{f(z) d z}{z-t}+\frac{1}{2 \pi i} \int_{\gamma} \frac{f(z) d z}{z-t}
$$

The second of these integrals vanishes, for $f(z) / z-t$ remains finite in the neighborhood of $c$, and when the radius of $\gamma$ approaches 0 , the length of the path of integration approaches 0 . But the value of the integral is constant. hence it must be 0 . Thus the formula

$$
f(t)=\frac{1}{2 \pi i} \int_{c} \frac{f(z) d z}{z-t}
$$

represents the function $f(t)$ at all points within $C$ except $c$. But the integral on the right hand side of this equation defines a function of $t$ that is analytic in $c$ too. Hence the theorem.

Second Proof. I now proceed to give a second elementary proof of $(A)$, making use of the definition of I. Let

$$
\varphi(z)=(z-c) f(z), \quad z \neq c ; \quad \varphi(c)=0 .
$$

Then $\varphi(z)$ is analytic at $c$ too; for $\varphi(z)$ is continuous throughout the region about $c$ and $\int \varphi(z) d z$ taken along any closed path of this region vanishes, as will now be shown. Integration by parts gives

$$
\text { where } \begin{aligned}
\int(z-c) f(z) d z & =(z-c) F(z)-\int F(z) d z \\
F(z) & =\int_{a}^{z} f(z) d z
\end{aligned}
$$


is continuous and as is readily seen single-valued. Thus the first term on the right taken along any closed path of the region is 0 . As to the second term, consider any closed path not including $c$. Then $F(z)$ and $F^{\prime}(z)=f(z)$ are both continuous throughout the enclosed region and hence the integral along the boundary of the region is $0 . *$ Finally let the path include $c$ and surround $c$ by a small circle. Then $\int F(z) d z$ extended in the usual way over the path and the circle will vanish. But the contribution that the circle yields is readily seen to be null; and thus $\int F(z) d z$ along any closed path whatever vanishes. Hence $\varphi(z)$ is analytic at $c$ too. Its derivative at $c$ is

$$
\lim _{z=c} \frac{\varphi(z)}{z-c}=\lim _{z=c} f(z)
$$

Hence $f(z)$ is continuous at $c$ and thus fulfills both conditions of the definition of an analytic function given in I, and the proof is complete. $\dagger$

HaRVARD UNIVERSITY, March 1896.

\section{ON THE MOTION OF A HOMOGENEOUS SPHERE OR SPHERICAL SHELL ON AN INCLINED PLANE, TAKING INTO ACCOUNT THE ROTATION OF THE EARTH.}

BY PROFESSOR ALEXANDRE S. CHESSIN.

The influence of the rotation of the earth on the motion of bodies on its surface has been the subject of most interesting experiments, of which those with falling bodies and those of Foucault with the pendulum and the gyroscop may be mentioned. We propose to give here another interesting illustration of that influence, namely, on the motion of a homogeneous sphere or spherical shell on a plane inclined to the horizon. We will identify the system of axes $(X, Y, Z)$ with the absolute, the system $(\Xi, r, Z)$ with the relative

* Cf. Goursat's proof of this theorem, Acta Math., vol. IV.; Harkness and Morley, Theory of Functions, p. 164.

+ Hölder has given in the Math. Annalen, vol. 20, 1882, a proof very similar to this. He integrates the given function $f(z)$ twice and shows that the second integral is analytic at $c$ too. Hence he infers that $f(z)$ is analytic at $c$. I had obtained the proof here given, however, before Hölder's proof was known to me. 